\title{
A Method of Target Detection in Remote Sensing Image Captured based for Sensor Network
}

\author{
Yingchun Shen ${ }^{\mathrm{a}, \mathrm{b}}$, Hai Jin ${ }^{\mathrm{a}}$ \\ ${ }^{a}$ School of Computer Science and Technology Huazhong University of Science and Technology, Wuhan, \\ 430074, China \\ ${ }^{b}$ Wuhan Digital Engineering Institute, Wuhan, 430074, China
}

\begin{abstract}
A refined energy constrained minimization method is developed for target detection in hyperspectral remote sensing images captured by unmanned aerial vehicles (UAVs) during their surveillance missions, which has been tested in the experiment under this paper. The experiment result proves, in the detection process, this method can effectively restrain noises so far as the spectral characteristics of any potential target are known, and find sub-pixel targets out effectively from the hyperspectral remote sensing image in unknown background spectrum.
\end{abstract}

Index Terms: sensor network; target detection; hyperspectral image; energy constrained minimization

(C) 2012 Published by MECS Publisher. Selection and/or peer review under responsibility of the Research Association of Modern Education and Computer Science

\section{Introduction}

One of major applications of sensor network technology is in UAVs' swarming combats. This paper presents a refined target detection method to find out targets from captured information by a sensor network formed by multiple UAVs performing optical remote sensing reconnaissance mission. In the experiment in this paper, the refined energy constrained minimization method is tested by using the data captured by UAVs' airborne hyperspectral imagers. The satisfied test result shows the method is capable in effectively solving the problem mentioned-above.

\section{Energy Constrained Minimization Method}

\subsection{Theory of Energy Constrained Minimization Method}

The energy constrained minimization method is proposed on the basis of linear mixing model [2].

Corresponding author:

E-mail address: hjin@hust.edu.cn 
We design a FIR filter: it has a weight vector $w=\left(\begin{array}{llll}w_{1} & w_{i} & w_{n}\end{array}\right)^{T}$ consisting of waveband number weights, regarding each element $R_{i}$ in the high dimensional data set $S=\left(\begin{array}{lll}R_{l} & R_{i} & R_{q}\end{array}\right)$ consisting of all pixels in the hyperspectral image to be detected. Multiply it to the said weight vector and obtain an output value:

$Y_{i}=w^{T} R_{i} \quad i=1, \ldots, q$

where, $q$ refers to the number of elements in the data set $S$, namely, the number of pixels in the hyperspectral image; $R_{i}=\left(\begin{array}{lll}r_{1} & r_{i} & r_{n}\end{array}\right)^{T}$ refers to the data vector of each pixel in the hyperspectral image to be detected, where $r_{i}$ refers to the reflection factor on the $i$ spectral band of the pixel, and $n$ refers to the number of waveband of the image.

Define the average output energy that is jointly determined by filter and data set $S$ as:

$E \square \frac{1}{q} \square_{i=1}^{q} y_{i}^{2} \square \frac{1}{q} w^{T} \square_{\square}^{\square} r_{i-1}^{q} r_{i}^{T} \square w \square w^{T} R w$

where, $R$ refers to the auto-correlative matrix of the data set $S$.

$R \square \frac{1}{q \square \square} \square_{i=1}^{q} y_{i}^{2} \square$

The energy constrained minimization method requires minimal energy of the output image processed by such filter. In addition, it requires that the output value (via weight vector multiplication) of target spectrum to be detected is 1 . So, the question resolves itself into the following:

$\operatorname{Min}\left\{w^{T} R w\right\} \quad$ s.t. $w^{T} d=1$

The spectrum $d$ of the target to be detected is given, and autocorrelative matrix $R$ can be obtained from data set $S$, therefore, the solution of above question can be obtained via optimization problem.

$w \square \frac{R^{-1} d}{d^{T} R^{-1} d}$

Each pixel $R_{i}$ in the hyperspectral image data set $S$ is calculated via this weight in sequence, thus obtaining a single band grey image, which can be used to distinguish the target from the background terrain features. In the image, due to the end member spectrum (except target spectrum) and noises are restrained, only pixels containing target spectrum have relatively larger DN values. In fact, the DN values of pixels containing target spectrum reflect the abundance [3] of target spectrum contained in these pixels. Hence, we can distinguish the target points from the background points through image binarization.

\subsection{Improvement on Energy Constrained Minimization Method}

In the equation (4), the inverse matrix $R^{-1}$ of autocorrelative matrix should be solved. In hyperspectral images, the condition number of autocorrelative matrix $R$ sometimes could be very large, becoming an ill-conditioned matrix. It is difficult to get correct result if using the method before refinement to solve the inverse matrix of such ill-conditioned matrix. For correct resolution, we adopt eigenvector decomposition method of eigenvalue of a matrix to solve the inverse matrix of autocorrelative matrix $R$.

The autocorrelative matrix $R$ can be decomposed as follows:

$R=V \cap V^{T}$

where, $\cap \operatorname{diag}\left(\begin{array}{lll}\lambda_{1} & \lambda_{i} & \lambda_{n}\end{array}\right)$ is an $n \times n$ diagonal matrix, $n$ refers to the waveband number of image, $\lambda_{i}$ refers to every eigenvalue of autocorrelative matrix $R$.

$V=\left(\begin{array}{llll}v_{1} & v_{i} & v_{n}\end{array}\right)$ is also an $n \times n$ matrix, in which each column matrix $v_{i}$ is an eigenvector corresponding to the $\lambda_{i}$. 
With respect to the detection of target points in wide distribution or large number, it has been proven [4] that certain maximal eigenvalues and their corresponding eigenvectors can be used to solve the inverse matrix of autocorrelative matrix. The number of eigenvalues used for problem solving is exactly the so-called intrinsic dimensionality. How to solve the intrinsic dimensionality becomes the key to tackle this problem. We adopt MNF (Minimum Noise Fraction Rotation) [5] method to solve the intrinsic dimensionality of image.

The first step for MNF analysis is de-correlation and recalibration of the image data based on covariance matrix of noise contribution, which makes the noise contribution to be featured by single variance and free from inter-band relativity. The second step is a standard principal component analysis on the new data obtained in the first step. Finally, divide the result images into two sections, namely, one with wavebands featuring large eigenvalues and principal components, and another with wavebands featuring small eigenvalues and major noise contributions via comparison of eigenvalues and corresponding result images. After MNF transformation, the wavebands, whose eigenvalues equal to 1 , present the images that primarily feature noises, while all of the eigenvalues of those wavebands that carry the principal components are greater than 1.

Based on these characteristics, the intrinsic dimensionality of image can be determined by the curve of MNF eigenvalues, as shown in Fig. 1. In Fig. 1, the intrinsic dimensionality of the image is determined as 10 according to the longitudinal coordinate values of the points whose MNF eigenvalues equal to 1 respectively.

If the intrinsic dimensionality is $p$, the autocorrelative matrix can be approximately decomposed as:

$$
R \square V \cdot V^{T}
$$

where, $\ldots \square \operatorname{diag}\left(\ell_{1} \ldots \ell_{i} \ldots \ell_{p}\right)$ is a $p \times p$ diagonal matrix, each $\lambda_{i}$ is one of the maximal $p$ eigenvalues of autocorrelative matrix $R . V \square\left(v_{1} \square \square v_{i} \square \square v_{p}\right)$ is an $n \times p$ matrix, and each column matrix $v_{i}$ is an eigenvector corresponding to $\lambda_{i}$. Therefore, find inverse solution of equation (6) out, and then the approximate solution of inverse matrix of autocorrelative matrix $R$ can be obtained as:

$R^{-1} \square V \cdots^{-1} V^{T}$

where, ${ }^{-1} \square \operatorname{diag}\left(\ell_{1}^{-1} \square \square \ell_{i}^{-1} \square \square \ell_{p}^{-1}\right)$

In this way, the ill-conditioned problem in high dimensional autocorrelative matrix inversion in (4) can be avoided accordingly.

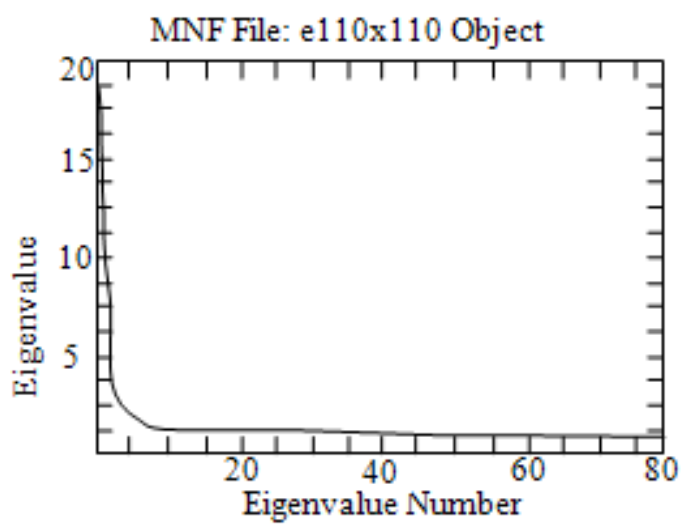

Figure 1. MNF Curve of Hyperspectral Remote Sensing Image 


\section{Experiment and Analysis}

\section{1 Data Description}

We firstly employ a UAV hyperspectral remote sensing image, which mainly consists of five types of terrain features -- oxidized basalt, rhyolite, bolson, shadow and vegetation as shown in Fig. 2. In the simulation experiment, an appropriate experimental image is generated by means of adding andradite target on certain points in the hyperspectral image so as to obtain sub-pixel targets.

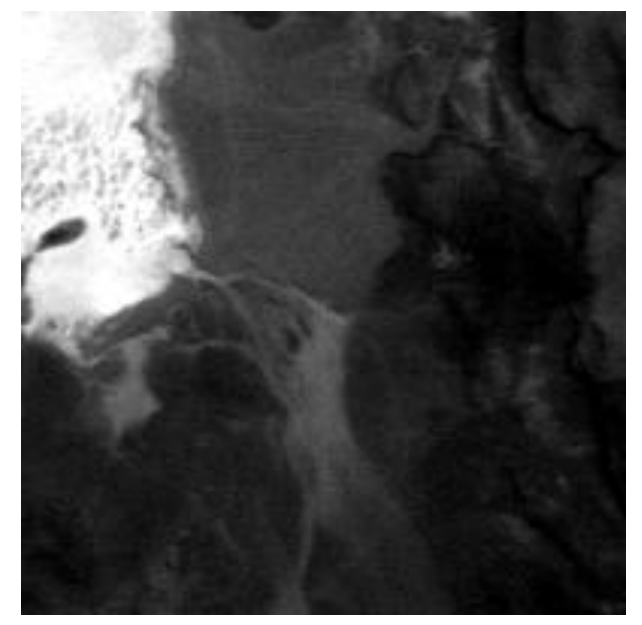

Figure 2. UAV Data

\section{2 Experimental Results and Analysis}

During the experiment, both the refined energy constrained minimization method and spectral angle matching (SAM) [6] method are adopted in target detection to the data images that contain target points generated by the means mentioned above. Fig. 3 and Fig. 4 are the detection outputs of the single band images by using these two methods respectively. Fig. 3 shows that, using the refined energy constrained minimization method, the output values corresponding to 50 target points are apparently greater than non-target points, say, closing to 1 . (Later, a threshold split process will separate the target points accordingly.) Fig. 4 shows that, using spectral angle matching method, only the output values of partial target pixels are relatively larger. Fig. 5 shows that all of the 50 targets are identified via the refined energy constrained minimization method, and there is only 1 false alarm target. The target detection rate is $100 \%$; the false alarm rate is as low as $2 \%$.

In the experiment, the threshold split is performed according to the requirements of constraint conditions under the energy constrained minimization method. The target points are those whose DN value is 1 in the output images. However, all 40 target points in the first 4 groups are sub-pixel's, and, in addition, contain background spectrum, it is impossible for the DN value of its relevant point in their corresponding output image to reach 1. However, in the output image, all of these sub-pixel target points that contain target spectrum have a relatively larger DN value. 


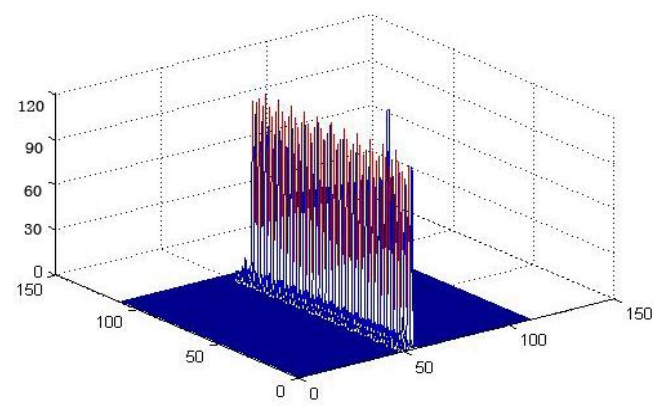

Figure 3. The Characteristic Graph After First Analog Data CEM Detection

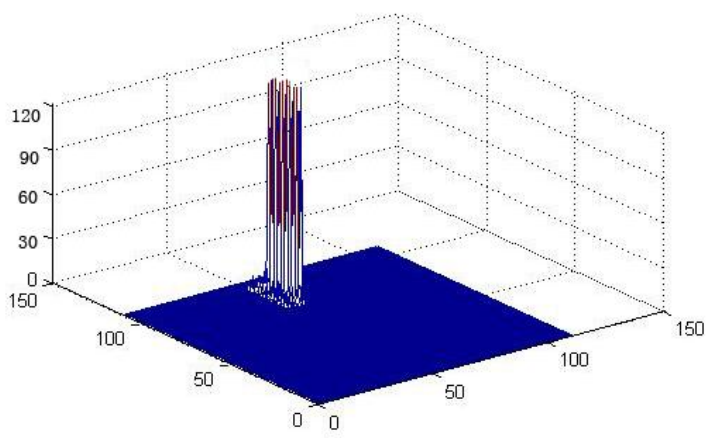

Figure 4. Characteristic Graph After First Analog Data SAM Detection

Based on this fact, the target points can be clearly distinguished via threshold split while the image passes the wave filter. This experiment shows that the refined energy constrained minimization method can find out all the pure pixel targets and sub-pixel targets, while spectral angle matching method cannot find out sub-pixel targets although it can accurately identify the 10 pure pixel targets in the fifth group. Meanwhile, the spectral angle matching method does not generate any false alarm target, because the spectral angle matching method detects target on the basis of strict matching of spectral characteristics, and assumes all points that do not strictly match with given target spectral characteristics as background points.

Fig. 5 and Fig. 6 respectively show the result images via threshold split process for output images after above detection. Fig. 6 shows that, if using spectral angle matching method, among 50 targets in the original 5 groups, only 10 pure pixel targets in the fifth group is detected, but 40 sub-pixel targets in the first 4 groups cannot be detected, therefore, the target detection rate is only $20 \%$. This experiment result indicates that although the target points are disorderly distributed, the refined energy constrained minimization method we developed still offers satisfactory results to the detection of sub-pixel targets, and is much better than spectral angle matching method. 


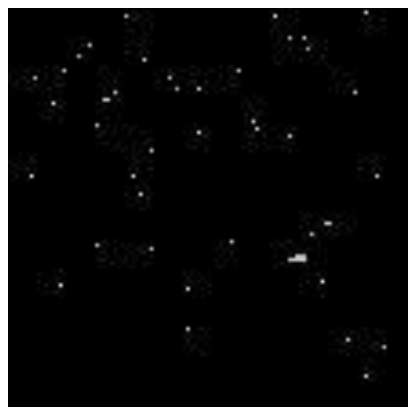

Figure 5. Threshold Split Graph after Second Analog Data CEM Detection

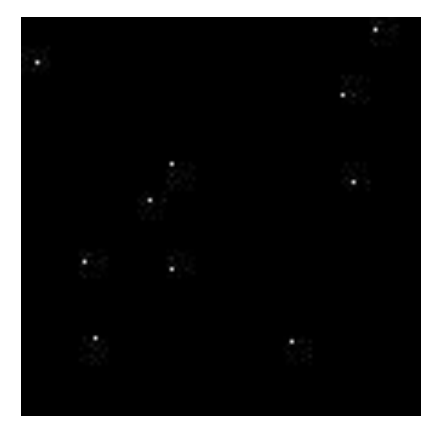

Figure 6. Threshold Split Graph after Second Analog Data SAM Detection

In Fig. 5, the target detection rate drops from $100 \%$ to $90 \%$ when employing the refined energy constrained minimization method on target detection to the second analog data image. It is because, in the second analog data image, target points are generated at random locations, background signals and noises may cause much more intensive interference to the target signals. Such situation is more similar to the actual situation. Therefore, the detection results are more convincible. In fact, the miss-detected 5 signals are ones with only $10 \%$ target pixels in the first group. Their target signals are extremely weak and are difficult to be separated from noises and background signals that are much stronger and more intensive. The fact also tells us that one of the preconditions to separate and quantify weak sub-pixel target by using the energy constrained minimization method is that the ${ }^{]}$ target signals are separable from noises and background signals [7].

\section{Conclusion}

The application of wireless sensor network concept to UAVs provides strong technical support to UAVs' swarming combat mode. Through defining FIR wave filter, the energy constrained minimization method can minimize the UAVs' surveillance image energy after wave filtration process under constraint conditions. This method effectively constrains non-target signals, especially noises in the output images. In addition, this method can separate reconnaissance target points from background points by application of constraint conditions which are satisfied only by target signals. This paper utilizes MNF method to solve intrinsic dimensionality, thus solving the ill-conditioned problems of high dimensional data that are difficult to be solved by using the energy constrained minimization method. In addition, we improve the method and extend its range of application. Experiment indicates that the method developed in this paper can achieve perfect results in detection of sub-pixel targets in the hyperspectral remote sensing image of unknown background spectrum. 


\section{References}

[1] J. Hill and D. Culler, "A Wireless Embedded Sensor Architecture for System-Level Optimization", Technical Report, U.C. Berkeley, 2001.

[2] J. C. Harsanyi, "Detection and classification of subpixel spectral signatures in hyperspectral image sequences", Ph.D. Dissertation, Department of Electrical Engineering, University of Maryland Baltimore County, Baltimore, MD, 1993.

[3] J. J. Settle and N. A. Drake, "Linear mixing and the estimation of ground cover proportions", Int. J. Remote Sensing, Vol.14, No.6, 1993, pp.1159-1177.

[4] C.-I Chang and D. C. Heinz, "Constrained Subpixel Target Detection for Remotely Sensed Imagery", IEEE Transactions on Geoscience and Remote Sensing, Vol.38, No.3, May 2000, pp.1144-1160.

[5] A. A. Green, M. D. Craig, and C. Shi, "The Application Of The Minimum Noise Fraction Transform To The Compression And Cleaning Of Hyper-spectral Remote Sensing Data", Proceedings of International Geoscience and Remote Sensing Symposium (IGARSS'88), Vol.3, Sep 1988, pp.1807-1807.

[6] R. A. Schowengerd, Remote sensing: models and methods for image processing, 2nd ed. Academic Press, 1997.

[7] J. Settle, "On Constrained Energy Minimization and the Partial Unmixing of Multispectral Images", IEEE Transactions on Geoscience and Remote Sensing, Vol.40, No.3, March 2002, pp.718-722. 